\title{
Lentivirus-delivered short hairpin RNA targeting SNAIL inhibits HepG2 cell growth
}

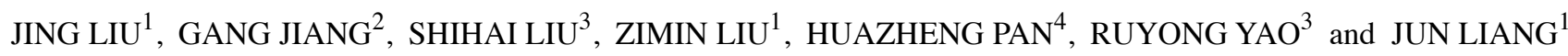 \\ Departments of ${ }^{1}$ Oncology, ${ }^{2}$ Radiology, ${ }^{3}$ Central Laboratory, and ${ }^{4}$ Department of Clinical Laboratory, \\ The Affiliated Hospital of the Medical College, Qingdao University, Qingdao 266003, P.R. China
}

Received January 27, 2013; Accepted April 9, 2013

DOI: $10.3892 /$ or.2013.2552

\begin{abstract}
Hepatocellular carcinoma (HCC) is the third leading cause of cancer-related mortality worldwide, and the highest incidence rates are reported in East Asia. We previously showed that SNAIL is upregulated in HCC tissues. In the present study, we aimed to investigate RNA interferencemediated targeting of SNAIL on the growth of HepG2 cells. We constructed three RNA interference plasmids targeting the SNAIL gene and selected the most efficient shRNA expression cassette. After the lentivirus (LV)-SNAIL small interfering (si)RNA vector was transfected into the HepG2 cell line, cell proliferation was measured using the MTT assay. E-cadherin mRNA and protein expression levels were examined by quantitative PCR and western blotting, respectively. We successfully constructed an LV-SNAIL siRNA lentiviral vector and demonstrated that it suppressed the expression of the SNAIL gene in HepG2 cells. RNA interference of SNAIL by the LV-SNAIL siRNA construct significantly inhibited the growth of HepG2 cells, in addition to significantly increasing E-cadherin mRNA and protein expression. Our findings strongly suggest that SNAIL and E-cadherin play a significant role in HCC progression, and exhibit a negative correlation. Furthermore, the expression of E-cadherin may be responsible for the reduced proliferation and survival of HepG2 cells. Thus, the SNAIL signaling pathway may provide a novel therapeutic target for the treatment of HCC.
\end{abstract}

\section{Introduction}

Hepatocellular carcinoma (HCC) is a common, highly invasive malignant tumor associated with a high mortality rate. It is the third leading cause of cancer-related mortality worldwide (1) and the highest incidence rates are reported in East Asia $(2,3)$. Recurrence, metastasis and the development of new primary

Correspondence to: Professor Jun Liang, Department of Oncology, The Affiliated Hospital of the Medical College, Qingdao University, 16 Jiangsu Road, Qingdao 266003, P.R. China

E-mail: liangjun1959@yahoo.com.cn

Key words: HepG2 cells, hepatocellular carcinoma, lentivirus, SNAIL, E-cadherin tumors are the most common causes of mortality among patients with HCC (4). The mainstay of therapy is surgical resection, however, only $10-20 \%$ of patients are suitable for surgical treatment (5). In addition, recent developments in genomic technologies have enabled the study of molecular aberrations and deregulations directly from patient specimens in a comprehensive manner (6). However, the target gene locus associated with genomic therapy remains unelucidated. Thus, given the single means of treatment and the lack of a targeted locus, there is an urgent need for developing novel treatments that can prevent the early invasion and metastasis of HCC and for surveying new molecular-targeted sites that offer the possibility for the effective development of gene therapy.

Epithelial to mesenchymal transition(EMT), which converts epithelial cells into migratory mesenchymal cells, is crucial for embryonic development. It is characterized by upregulation of extracellular matrix components, loss of intercellular cohesion, an increased rate of cellular migration and invasion, as well as increased resistance to apoptosis. Recently, EMT has emerged as a pivotal event in the development of invasion and metastasis associated with cancer progression (7). This type of transition involves a decrease in E-cadherin expression and gain of vimentin expression, leading to increased cell migration, invasion and tumorigenicity (8). Downregulation of E-cadherin is a major event in EMT. E-cadherin, which is a key cadherin, plays a major role in the establishment and maintenance of intercellular adhesion, cell polarity and tissue architecture (9). Currently, there is substantial evidence that functional perturbation of E-cadherin expression during the process of EMT occurs at the transcriptional level. According to studies, numerous transcription factors have been associated with the regulation of EMT, including SNAIL (10), Zeb1 (11), Zeb (2), Twist1 (12) and Slug (13). Among these regulators, SNAIL is a prominent inducer of EMT which strongly suppresses E-cadherin expression (14).

SNAIL is a well-known zinc finger $(\mathrm{ZF})$ transcriptional repressor which is essential during embryonic development (15). Numerous studies have shown that SNAIL is significantly upregulated in a variety of malignant tumors. There is also evidence that SNAIL enhances invasive and metastatic potential, and the malignant tumors exibiting SNAIL upregulation are prone to distant metastasis, deep invasion and poor prognosis. Conversely, in vitro experiments have shown that inhibition of SNAIL mRNA expression significantly reduces 


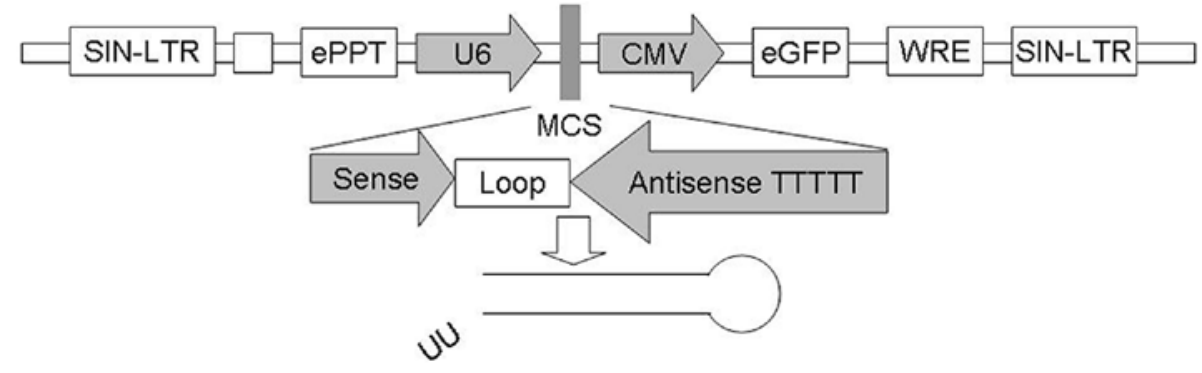

Figure 1. Scheme of shRNA production mediated by lentiviral vectors.

cell motility and invasion capacity and decreases invasion to the extracellular matrix (16). Briefly, SNAIL-induced EMT converts epithelial cells into mesenchymal cells with migratory properties contributing to the acquisition of invasive and metastatic potential of tumors. In addition, the induction is largely due to the repression of E-cadherin. Thus, E-cadherin repressors are regarded as markers of malignancy and metastasis and provide hope for gene therapy of HCC. However, the molecular mechanism of SNAIL in regulating HCC carcinogenesis and progression remains unclear. In the present study, we verify the relationship of SNAIL and E-cadherin in vitro by the RNA interference (siRNA) method.

Small hairpin RNA (shRNA) expression vector systems have been established to induce RNA interference (RNAi) in mammalian cells (17). RNAi has emerged as a powerful technique through which to downregulate the expression of specific genes in cells and in animals. Among the shRNA delivery mechanisms, viral vectors, and in particular lentiviral vectors, are capable of specific, highly stable and functional silencing of gene expression in a variety of human cells including primary non-dividing cells (18). In the present study, we used a lentiviral vector, carrying SNAIL shRNA for transfection into HepG2 cells in order to knock down the expression of the target gene SNAIL. Through gene expression analysis at the mRNA and protein levels, we observed that knockdown of SNAIL led to the activation of E-cadherin expression, resulting in reduced cell proliferation and viability of HCC cells. Thus, targeted inactivation of SNAIL may provide a novel therapeutic strategy for $\mathrm{HCC}$ patients expressing this gene.

\section{Materials and methods}

Construction of the pGenesil-SNAIL siRNA. Three pairs of shRNAs were used for screening the most effective downregulation of the gene fragment. In the enzyme site of the vector pGenesil-1, three coding regions corresponding to the target human SNAIL (NM_005985), starting at positions 122, 150 and 518 in the sequence were selected as siRNA target sequences under the guide of the siRNA designing software. The target shRNA oligonucleotides were annealed and ligated with the siRNAs, and the pGenesil-1 vector was cleaved by BamHI and HindIII, and then the products were recovered and purified. shRNA oligonucleotide fragment and the pGenesil-1 vector were ligated, and the recombinant plasmids were named pGenesilSNAIL shRNA-1-3. Next, pGenesil-GFP-SNAIL shRNAs were transfected into HepG2 cells, respectively, using the Lipofectamine 2000 reagent (Invitrogen, Carlsbad, CA, USA). The most effective siRNA, by screening using analysis of the SNAIL mRNA and protein levels in HepG2 cells, was detected and used for subsequent experiments.

Construction and production of lentiviral vectors (Fig. 1). The most effective shRNA expression cassette was selected and cut off by BamHI and MluI from the pGenesil-1 vector, and ligated into pLV-mCMV-ZsGreen-PGK-puro shuttle vector, which was called pLV-SNAIL shRNA. Plasmids were purified with a MaxPrep kit and successful ligations were verified by sequencing (Sango, China). Recombinant lentiviral vectors were produced by co-transfecting HEK-293T cells with the lentiviral expression plasmid and packaging plasmids using the calcium phosphate method. Briefly, $8 \mu \mathrm{g}$ shRNA plasmid DNA, $5 \mu \mathrm{g}$ lentiviral helper1 and $6 \mu \mathrm{g}$ lentiviral helper 2 plasmids were mixed with sterile $\mathrm{ddH}_{2} \mathrm{O}$ to a final volume of $450 \mu \mathrm{l}$ and mixed with $50 \mu \mathrm{l}$ of $2.5 \mathrm{M} \mathrm{CaCl}_{2}$. After transfection, infectious media, containing shRNA lentiviral vectors, were harvested at 48 and $72 \mathrm{~h}$.

Cell culture and infection. The human hepatocellular cancer HepG2 cell line and HEK293T cells were purchased from the American Type Culture Collection (ATCC, Manassas, VA, USA). Cells were grown in $5 \% \mathrm{CO}_{2}$ at a saturated humidity, at $37^{\circ} \mathrm{C}$ and cultured as a monolayer in RPMI-1640 medium supplemented with $100 \mathrm{U} / \mathrm{ml}$ penicillin and $100 \mu \mathrm{g} / \mathrm{ml}$ streptomycin, $2 \mathrm{mmol} / \mathrm{l}$ glutamine and 10\% FBS.

HepG 2 cells were passaged to $40 \%$ confluence the following day. The viral media were added to HepG2 cells with $8 \mu \mathrm{g} / \mathrm{ml}$ Polybrene 4 times over two days. After infection for $72 \mathrm{~h}$, viral media were removed and cells were collected for further experiments.

Cell proliferation assay. Cell viability was assessed by the 3-(4,5-dimethylthiazol-2-yl)-2,5-diphenyltetrazolium bromide (MTT) method. HepG2 cells (the LV-SNAIL siRNA, LV-nonsense RNA and parental cell groups) were seeded in 96-well plates in culture medium at an optimal density $\left(5 \times 10^{3}\right.$ cells/well) in triplicate wells. After growing for 12, 24 and $48 \mathrm{~h}, 20 \mu \mathrm{l}$ of $5 \mathrm{mg} / \mathrm{ml}$ MTT (in PBS) was added to each well and continually incubated for $4 \mathrm{~h}$ at $37^{\circ} \mathrm{C}$ in a $\mathrm{CO}_{2}$ incubator. The formazan granules obtained from the cells were dissolved in $150 \mu \mathrm{l}$ dimethyl sulfoxide (DMSO) for $10 \mathrm{~min}$. Cell viability was then measured in terms of the optical density (OD) at a wavelength of $490 \mathrm{~nm}$. Each cell viability assay was performed and repeated three times. 

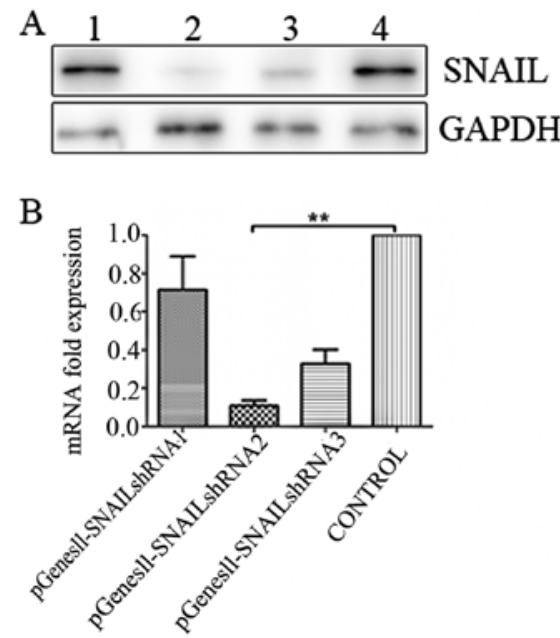

Figure 2. Effect of SNAIL shRNA expression. HepG2 cells were transfected with pGenesil vector harboring the shRNA targeting SNAIL. (A) Western blot analysis of SNAIL expression. Lane 1, HepG2 cells transfected with pGenesil-SNAIL shRNA1; lane 2, HepG2 cells transfected with pGenesilSNAIL shRNA2; lane 3, HepG2 cells transfected with pGenesil-SNAIL shRNA3; lane 4, HepG2 cells. (B) SNAIL mRNA expression as detection by real-time PCR. ${ }^{* *} \mathrm{P}<0.05$ vs. control.

Quantification by real-time PCR. Transfected and untreated cells were collected and washed with phosphate-buffered saline (PBS). Total RNA was extracted from the cells of the three groups, and cDNA was generated with a PrimeScript ${ }^{\circledR}$ RT reagent kit (Takara, Chiga, Japan) at a total volume of $20 \mu \mathrm{l}$ according to the manufacturer's instructions. cDNA was then used in each amplification reaction. Reactions were performed using the SYBR ${ }^{\circledR}$ Premix Ex Taq ${ }^{\mathrm{TM}}$ (Takara), under the following PCR conditions: $95^{\circ} \mathrm{C}$ for $30 \mathrm{sec}$, followed by 40 cycles of $95^{\circ} \mathrm{C}$ for $5 \mathrm{sec}$ and $60^{\circ} \mathrm{C}$ for $30 \mathrm{sec}$. Each sample was also subjected to melting curve analysis to confirm amplification specificity. GAPDH was used as a control housekeeping gene. The expression of SNAIL was assessed by normalization of the cycle threshold $(\mathrm{Ct})$ of these genes to that of GAPDH. A Ct value was obtained from each amplification curve by using the software provided by the manufacturer (Roche, Mannheim, Germany).

Western blot analysis. Cell lysates of the lentiviral-infected HepG2 cell clones and the non-transfected HepG2 cell controls were harvested and subjected to SDS-PAGE in $10 \%$ polyacrylamide gel. Bands were probed for SNAIL protein expression using rabbit polyclonal anti-SNAIL and secondary goat anti-rabbit alkaline phosphatase antibody (Abcam, Cambridge, MA, USA). GAPDH detection was performed using rabbit polyclonal anti-GAPDH and mouse monoclonal anti-GAPDH (Abcam). Blots were incubated with appropriate HRP-conjugated secondary antibodies and specific binding was detected by ECL (Pierce, Rockford, IL, USA).

\section{Results}

Selection of the most effective SNAIL-specific shRNA. Expression levels of SNAIL mRNA and protein in the HepG2 cell line were detected in previously published data by our laboratory (19). The levels of SNAIL mRNA and protein were

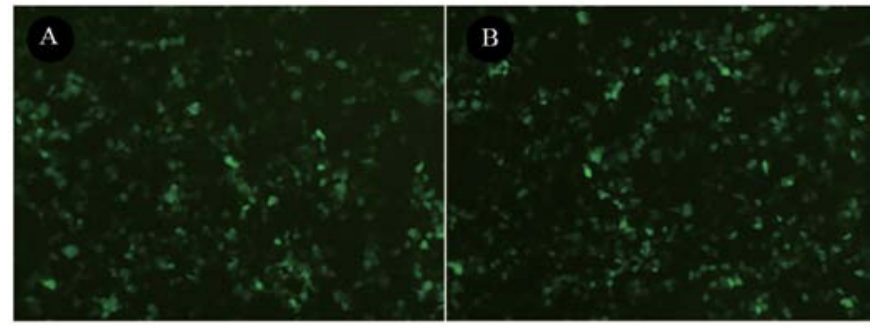

Figure 3. Verification of SNAIL knockdown in HepG2 cells by lentiviralmediated RNA interference. Images of GFP expression showing shRNA delivery efficiency. (Original magnification, x200). (A) LV-SNAIL shRNAinfected HepG2 cells; (B) LV-CONTROL-GFP-infected HepG2 cells.

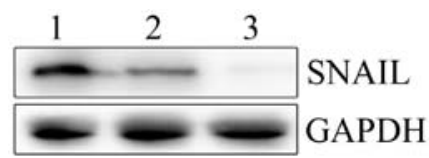

Figure 4. Lentiviral vector-mediated delivery of siRNA targeting SNAIL results in specific knockdown of expression. The indicated lentiviral siRNA expression constructs were cotransfected into $293 \mathrm{~T}$ cells with an expression construct for the SNAIL target. Immunoblotting of whole cell extracts was performed with anti-SNAIL antibody to detect SNAIL (upper panel). An anti-GAPDH antibody was used to confirm equal protein loading (bottom panel). Lane 1, blank HepG2 cells; lane 2, LV-CONTROL-GFP-infected HepG2 cells; lane 3, LV-SNAIL shRNA-infected HepG2 cells.

were previously found to be increased in metastatic tumor samples in comparison to non-metastatic tumor samples and the same phenomena were detected in HepG2 cells (19). We determined the effects of SNAIL inhibition on the viability and growth of HepG2 cells. For screening the most effective shRNA fragment, three plasmids containing shSNAIL (pGenesil-SNAIL shRNA) were constructed and transfected into HepG2 cells using the Lipofectamine 2000 reagent, respectively. GFP expression in the HepG2 cells was observed under a fluorescence microscope $36 \mathrm{~h}$ after transfection with the pGenesil vectors. Results of the western blot assay showed that pGenesil-SNAIL shRNA2 significantly suppressed the expression of SNAIL at the protein level in HepG2 cells, while real-time PCR determined expression at the mRNA level. According to the results of the real-time PCR and western blot assay, SNAIL shRNA2 was the most effective and, thus, was used in the subsequent experiments (Fig. 2).

Construction and analysis of the LV-SNAIL shRNA. The most efficient shRNA expression cassette was selected and constructed into the lentiviral vector, named LV-SNAIL shRNA. To determine the effect of LV-SNAIL shRNA on the expression of SNAIL, GFP expression was observed under a fluorescence microscope in the HepG 2 cells $72 \mathrm{~h}$ after infection with LV-SNAIL shRNA and LV-CONTROL-GFP (Fig. 3). Next, real-time PCR and western blotting were performed to determine the mRNA and protein levels of SNAIL in the LV-SNAIL shRNA, LV-CONTROL-GFP and parental cell groups. As shown in Fig. 4, LV-SNAIL shRNA significantly inhibited expression of SNAIL protein when compared with the levels in the HepG2 cells and the LV-CONTROL-GFP group. 


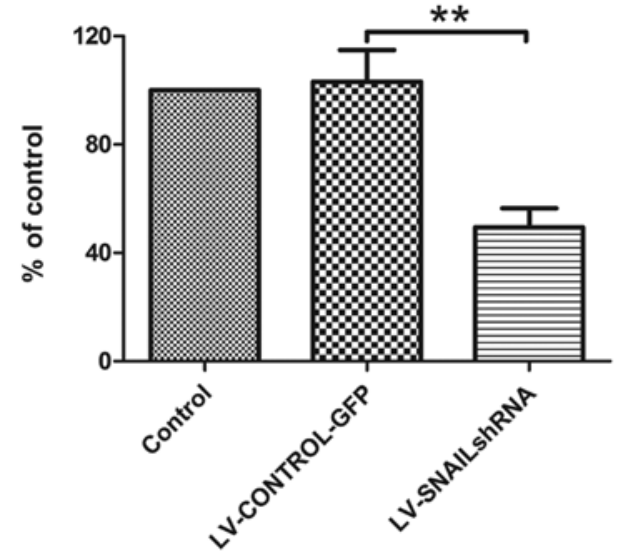

Figure 5. Effect of SNAIL knockdown in the HepG2 cell groups on cell proliferation as examined by MTT assay. ${ }^{* *} \mathrm{P}<0.05$ vs. control.

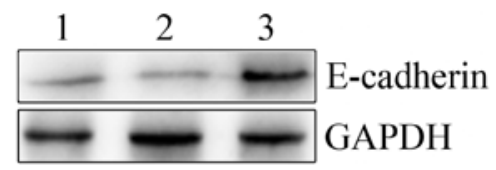

Figure 6. Expression of E-cadherin in HepG2 cell lines transfected with LV-SNAIL shRNA. HepG2 cells with low SNAIL expression exhibited upregulated E-cadherin expression.

Cell proliferation analysis in HepG2 cells. Cell proliferation was monitored for 4 days after HepG2 cells were infected with LV-SNAIL shRNA, LV-CONTROL-GFP and in parental cells without infection. MTT assay was used to evaluate cell viability. The absorption value at $490 \mathrm{~nm}$ was determined. As a result, we found that transfection with LV-CONTROL-GFP had no effect on the cell proliferation and viability in the cell groups (Fig. 5) while transfection with LV-SNAIL shRNA caused a dramatic reduction in proliferation when compared with the proliferation rate in the parental HepG2 cells.

E-cadherin expression assay. As shown in Fig. 6, E-cadherin protein expression was evaluated by western blotting in the human HepG2 cell lines. Silencing of SNAIL expression by LV-SNAIL shRNA significantly increased the expression of E-cadherin when compared with the LV-SNAIL shRNA and parent groups. LV-SNAIL shRNA markedly downregulated the protein level of SNAIL and upregulated the protein level of E-cadherin in HepG2 cells when compared with these levels in the other two cell groups.

\section{Discussion}

The SNAIL signaling pathway plays a significant role in various physiological processes, including cell growth, cell cycle regulation (20) and embryonic development (15). However, SNAIL has been implicated in cell proliferation, invasion and metastasis in a diverse group of human cancers, including liver cancer. Recent studies have also revealed that the promotion of SNAIL activation contributes to oncogenesis and the invasion and metastasis of cancers $(21,22)$. Thus, the purpose of the present study was to assess the changes in the
mRNA and protein expression of SNAIL and E-cadherin involved in EMT in HepG2 cell lines.

Epithelial to mesenchymal transition (EMT), which converts epithelial cells into migratory mesenchymal cells, is crucial for embryonic development. It is characterized by upregulation of extracellular matrix components, loss of intercellular cohesion, increased rate of cellular migration and invasion, as well as increased resistance to apoptosis. Recently, EMT has emerged as a pivotal event in the development of the invasive and metastatic potential associated with cancer progression (7). This type of transition occurs through a decrease in E-cadherin expression and gain of vimentin expression, leading to increased cell migration, invasion and tumorigenicity (8). Downregulation of E-cadherin is a major event in EMT. E-cadherin, which is a key cadherin, plays a major role in the establishment and maintenance of intercellular adhesion, cell polarity and tissue architecture (9). At present, there is substantial evidence that functional perturbation of E-cadherin expression during the process of EMT occurs at the transcriptional level. According to various studies, numerous transcription factors have been associated with regulation of EMT, including SNAIL (10), Zeb1 (11), Zeb (2), Twist1 (12) and Slug (13). Among these regulators, SNAIL is a prominent inducer of EMT which strongly suppresses E-cadherin expression (14).

In order to elucidate the role played by SNAIL in HCC, we suppressed SNAIL in HCC cells using the RNAi method by a lentiviral vector. RNAi is the process by which double-strand RNA induces potent and specific inhibition of eukaryotic gene expression through the degradation of complementary messenger RNA, and is functionally similar to the process of post-transcriptional gene silencing. The inhibitory potency and the method of transferring siRNA into HCC cells are two critical factors for successful application of the RNAi method. In recent years, shRNAs have been proven to provide longlasting silencing and maximal inhibition of gene expression at lower concentration (23). However, the inhibitory potency of shRNA is related to specificity of the target sequence, thus we used three pairs of shRNAs for screening the most effective downregulated gene fragment. Thus, we chose SNAIL siRNA2 which was able to knock out the expression of SNAIL efficiently. In addition, in order to identify the target gene or the vector fragment, we used EGFP siRNA as the control group and parental cells as the blank group. In the present study, we found that LV-SNAIL siRNA2 suppressed the expression of SNAIL at both the mRNA and protein levels in HCC. In the control group of HepG2 cells, however, the expression SNAIL was significantly increased compared with the experimental group. We concluded that LV-shSNAIL had a high specificity for SNAIL.

In the present study, a lentiviral vector was selected as our shRNA delivery vehicle since lentiviruses can transfect both dividing and non-dividing cells at a high efficiency and sustain long-term gene expression by integration into the host genome. What is more, lentiviral vectors are safe for use in humans, and they have the potential to become the means for human gene therapy. At present, an increasing number of studies suggest that activation of SNAIL plays an important role in the early metastasis of carcinomas (24). Furthermore, disruption of SNAIL expression has been reported to suppress 
cell metastasis by decreasing EMT and increasing epitheliumspecific genes, such as E-cadherin in other carcinomas (25). In our study, the expression of SNAIL and E-cadherin exhibited a negative correlation, which may be another major verification for our findings. Thus, our results indicate that blocking SNAIL expression upregulates E-cadherin expression directly or indirectly in HepG2 cells.

An increasing number of studies suggest that activation of SNAIL plays an important role in many carcinomas $(26,27)$. Furthermore, disruption of SNAIL at the transcriptional level has been reported to suppress cell invasion by decreasing cell-cell homotypic adhesion and increasing cell motility (14). Moreover, inappropriate and constitutive activation of SNAIL may be responsible for HCC progression by regulating expression of target genes, such as cadherin and MMP-2 (9). In the present study, the survival of cells using a cell proliferation analysis was examined and we found that silencing of SNAIL by RNAi in HepG2 cells resulted in reduced proliferation and survival. Conversely, the expression of E-cadherin in the SNAIL-silenced cell group was upregulated, which indicates that the reduced proliferation and survival which resulted from the silencing of SNAIL by RNAi was caused by the upregulation of the expression of E-cadherin.

Overall, our study indicates that siRNA targeting of SNAIL mRNA via a lentiviral vector system effectively sustains knockdown of the SNAIL gene expression in HepG2 cells. In the present study, we describe the successful construction of a lentiviral RNAi vector targeting SNAIL that may provide a useful tool with which to study the function of the SNAIL gene in liver cancer cells. Our findings strongly suggest that SNAIL and E-cadherin play a significant role in HCC progression, and exhibit a negative correlation. Furthermore, the expression of E-cadherin may be responsible for the reduced proliferation and survival of HepG2 cells. Targeting SNAIL activation may prove to be an effective approach for controlling HCC progression. Thus, the SNAIL signaling pathway may provide a novel therapeutic target for the treatment of HCC.

\section{References}

1. Parkin DM, Bray F, Ferlay J and Pisani P: Estimating the world cancer burden: Globocan 2000. Int J Cancer 94: 153-156, 2001.

2. Comijn J, Berx G, Vermassen P, et al: The two-handed E box binding zinc finger protein SIP1 downregulates E-cadherin and induces invasion. Mol Cell 7: 1267-1278, 2001.

3. Osada T, Sakamoto M, Ino Y, Iwamatsu A, Matsuno Y, Muto T and Hirohashi S: E-cadherin is involved in the intrahepatic metastasis of hepatocellular carcinoma. Hepatology 24: 1460-1467, 1996.

4. Tung-Ping Poon R, Fan ST and Wong J: Risk factors, prevention, and management of postoperative recurrence after resection of hepatocellular carcinoma. Ann Surg 232: 10-24, 2000.

5. Clark HP, Carson WF, Kavanagh PV, Ho CP, Shen $\mathrm{P}$ and Zaqoria RJ: Staging and current treatment of hepatocellular carcinoma. Radiographics 25 (Suppl 1): S3-S23, 2005.

6. Hoshida Y, Moeini A, Alsinet C, Kojima K and Villanueva A: Gene signatures in the management of hepatocellular carcinoma Semin Oncol 39: 473-485, 2012.

7. Hugo H, Ackland ML, Blick T, Lawrence MG, Clements JA, Williams ED and Thompson EW: Epithelial-mesenchymal and mesenchymal-epithelial transitions in carcinoma progression. J Cell Physiol 213: 374-383, 2007.
8. Neal CL, McKeithen D and Odero-Marah VA: Snail negatively regulates cell adhesion to extracellular matrix and integrin expression via the MAPK pathway in prostate cancer cells. Cell Adh Migr 5: 249-257, 2011.

9. Miyoshi A, Kitajima Y, Sumi K, Sato K, Haqiwara A, Koqa Y and Mivazaki K: Snail and SIP1 increase cancer invasion by upregulating MMP family in hepatocellular carcinoma cells. $\mathrm{Br}$ J Cancer 90: 1265-1273, 2004.

10. Cano A, Perez-Moreno MA, Rodriqo I, et al: The transcription factor Snail controls epithelial-mesenchymal transitions by repressing E-cadherin expression. Nat Cell Biol 2: 76-83, 2000.

11. Eger A, Aiqner K, Sondereqqer, et al: DeltaEF1 is a transcriptional repressor of E-cadherin and regulates epithelial plasticity in breast cancer cells. Oncogene 24: 2375-2385, 2005.

12. Yang J, Mani SA, Donaher JL, et al: Twist, a master regulator of morphogenesis, plays an essential role in tumor metastasis. Cell 117: 927-939, 2004.

13. Bolos V, Peinado H, Perez-Moreno MA, Fraga MF, Esteller M and Cana A: The transcription factor Slug represses E-cadherin expression and induces epithelial to mesenchymal transitions: a comparison with Snail and E47 repressors. J Cell Sci 116: 499-511, 2003.

14. Haraguchi M: The role of the transcriptional regulator snail in cell detachment, reattachment and migration. Cell Adh Migr 3: 259-263, 2009.

15. Peinado H, Olmeda D and Cano A: Snail, Zeb and bHLH factors in tumour progression: an alliance against the epithelial phenotype? Nat Rev Cancer 7: 415-428, 2007.

16. Palmer MB, Majumder P, Green MR, Wade PA and Boss JM: A 3' enhancer controls Snail expression in melanoma cells. Cancer Res 67: 6113-6120, 2007.

17. Brummelkamp TR, Bernards R and Agami R: A system for stable expression of short interfering RNAs in mammalian cells. Science 296: 550-553, 2002.

18. Nishitsuji H, Ikeda T, Miyoshi H, Ohashi T, Kannaqi M and Masuda T: Expression of small hairpin RNA by lentivirus-based vector confers efficient and stable gene-suppression of HIV-1 on human cells including primary non-dividing cells. Microbes Infect 6: 76-85, 2004.

19. Chen D, Zheng X, Jiao X, Gao Y, Zhang K and Liang J: Transcriptional repressor snail and metastasis in hepatocellular carcinoma. Hepatogastroenterology 59: 1359-1365, 2012.

20. Park JH, Sung IJ, Lee SW, Kim KW, Kim YS and Yoo MA: The zinc-finger transcription factor Snail downregulates proliferating cell nuclear antigen expression in colorectal carcinoma cells. Int J Oncol 26: 1541-1547, 2005.

21. Fan XJ, Wan XB, Yang ZL, et al: Snail promotes lymph node metastasis and Twist enhances tumor deposit formation through epithelial-mesenchymal transition in colorectal cancer. Hum Pathol 44: 173-180, 2012.

22. Sun M, Guo X, Qian X, et al: Activation of the ATM-Snail pathway promotes breast cancer metastasis. J Mol Cell Biol 4: 304-315, 2012.

23. Kim DH, Behlke MA, Rose SD, Chang MS, Choi S and Rossi JJ: Synthetic dsRNA Dicer substrates enhance RNAi potency and efficacy. Nat Biotechnol 23: 222-226, 2005.

24. Emadi Baygi M, Soheili ZS, Schmitz I, Sameie S and Schulz WA: Snail regulates cell survival and inhibits cellular senescence in human metastatic prostate cancer cell lines. Cell Biol Toxicol 26: 553-567, 2010.

25. Becker KF, Rosivatz E, Blechschmidt K, Kremmer E, Sarbia M and Hofler H: Analysis of the E-cadherin repressor Snail in primary human cancers. Cells Tissues Organs 185: 204-212, 2007.

26. Merikallio H, Turpeenniemi-Hujanen T, Paakko P, et al: Snail promotes an invasive phenotype in lung carcinoma. Respir Res 13: 104, 2012.

27. Boutet A, Esteban M, Maxwell P and Nieto M: Reactivation of Snail genes in renal fibrosis and carcinomas: a process of reversed embryogenesis? Cell Cycle 6: 638-642, 2007. 